\section{Chemoenzymatic preparation of germacrene analogues $\dagger$}

\author{
Oscar Cascón, Sabrina Touchet, David J. Miller, Veronica Gonzalez, Juan A. Faraldos and \\ Rudolf K. Allemann*
}

Received 31st July 2012, Accepted 14th August 2012

DOI: $10.1039 / \mathrm{c} 2 \mathrm{cc} 35542 \mathrm{f}$

A small library of novel germacrenes was generated using a combination of two plant enzymes, germacrene A synthase, and D synthase and modified farnesyl diphosphate (FDP) analogues. This chemoenzymatic approach allows the preparation of potentially valuable volatiles for biological studies.

Terpenoids represent a valuable class of bioactive fine chemicals ${ }^{1}$ and are therefore attractive targets for synthetic modification; modulation of their natural properties may lead to new medicinal and agrochemical compounds with improved properties. However, the complexity of the hydrocarbon skeletons and the often significant chemical instability of many terpenoids can present a formidable challenge to the synthetic chemist. ${ }^{2}$ Synthetic biology approaches have focused on the preparation of natural terpenoids in living organisms, ${ }^{3 a}$ but they operate with whole biochemical pathways using fundamental biosynthetic building blocks (e.g. isopentenyl diphosphate) and can therefore not easily be applied to generate modified terpenes. One attractive synthetic approach that complements current terpene synthetic biology ${ }^{3 b, c}$ and circumvents the difficult task of engineering full metabolic pathways to generate alternative substrates in vivo, could rely on the chemical preparation of FDP analogues as substrates of recombinant terpene synthases to create modified terpenoids. Modified FDPs ${ }^{4,5}$ have been used extensively to study the mechanisms of the cationic reactions mediated by (sesqui)terpene synthases. ${ }^{4-7}$ However, despite the fact that several unnatural FDPs are indeed turned over by these enzymes, ${ }^{4,5,8}$ only a few reports have explored the synthetic utility of terpene synthases toward the production of valuable novel terpenoids. ${ }^{9}$

Germacrene A and germacrene D synthases (GAS and GDS) are two plant sesquiterpene synthases that catalyze the $\mathrm{Mg}^{2+}$-dependent conversion of FDP (1a) to germacrene A (3a) and germacrene D (5a), respectively (Fig. 1). These two macrocyclic sesquiterpenes have been shown to act as semiochemicals affecting the olfactory response of insects. ${ }^{10}$ While a synthesis of the rather unstable germacrene D (5a) has been reported, ${ }^{11}$ the extreme thermal and photochemical instability

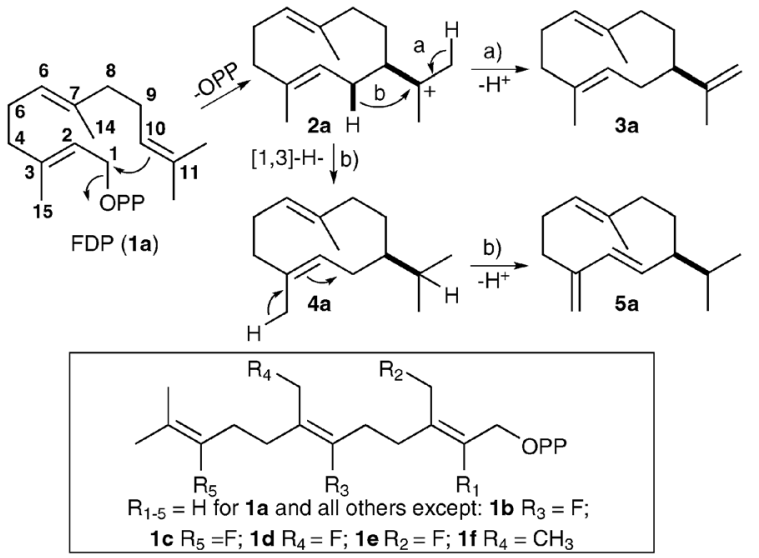

Fig. 1 Proposed biosynthesis of germacrenes A (3a) and D (5a). Modified substrate analogues of GAS and GDS (framed).

of the acid labile germacrene A (3a) has so far hampered the development of a satisfactory chemical synthesis. ${ }^{12}$ Nevertheless, fluorinated germacrene A analogues with improved stabilities have previously been produced enzymatically from fluorinated FDP analogues. ${ }^{4 a, c, 5 b}$

Thus, based on the biological and potential economic significance of compounds 3a and 5a, germacrene A and D synthases from Solidago canadensis ${ }^{13}$ were selected to investigate their capability to produce non-natural germacrenes from modified FDPs. To this end, recombinant GAS and GDS were overproduced in E. coli and purified as previously described. ${ }^{9 b, 14}$ Several fluorine and methyl modified FDPs were screened by GC-MS on an analytical scale for substrate activity. Germacrene A analogues were readily identified through their ability to undergo thermal Cope rearrangements to the corresponding $\beta$-elemene analogues under GC-MS conditions; ${ }^{14}$ germacrene D analogues were identified from their mass spectra since the presence of the more stable (i.e. more abundant, $100 \%)[\mathrm{M}-43]^{+}$fragment in the $\mathrm{EI}^{+}$-MS is diagnostic of the parent 5a. Only modified FDP analogues (Fig. 1, framed) that gave a relatively strong ion count in the total ion chromatogram (GC-MS) as compared with the natural substrate 1a were considered suitable for this study (vide infra).

Interestingly, in contrast to what has been observed with other sesquiterpene synthases, ${ }^{4 a, d, 9 a}$ 2-fluoro-FDP was not turned over significantly by GAS or GDS. In addition, the $\mathrm{H} / \mathrm{F}$ and in particular the $\mathrm{H} / \mathrm{CH}_{3}$ substitution at the $\mathrm{C} 15$ position of FDP 
was not tolerated by either enzyme. The full details and the mechanistic implications of these observations are beyond the scope of this manuscript and will be published elsewhere.

Optimal reaction conditions for the preparation of modified germacrenes: initial preparative incubations using both enzymes were shown to be inefficient and hence an optimisation of the reaction conditions was carried out. After some experimentation, conversions were found to be optimal at concentrations of $\mathrm{Mg}^{2+}$, FDP and enzyme of $10 \mathrm{mM}$ (5 $\mathrm{mM}$ for GAS), $0.35 \mathrm{mM}$ and $6 \mu \mathrm{M}$, respectively (ESI $\dagger$ ). Higher concentrations of $\mathrm{Mg}^{2+}$ and/or enzyme led to the formation of insoluble/ inactive $\mathrm{FDP}-\mathrm{Mg}^{2+}$-complexes and/or enzyme aggregation, which in turn resulted in less efficient turnovers. The concentration of GDS (but not GAS) could be increased to $12 \mu \mathrm{M}$ simply by inclusion of $1 \%$ of the non-denaturing detergent 3-[(3-cholamidopropyl)dimethylammonio]-1-propanesulfonate) (CHAPS) in the assay buffer. The nature of the organic solvent, the reaction vessel itself and the extractive work up were of fundamental importance for optimal conversions. ${ }^{9 b}$ In the final optimised conditions, $d$-chloroform was used as the organic layer, the incubations were carried out in sealed tubes with gentle agitation and enzymatic products were extracted overnight using an automated rotator. The filtered and dried $d$-chloroform solutions were then analysed by GC-MS and NMR-spectroscopy. Under these conditions, the enzymatic conversions of $\mathbf{1 a}$ to germacrene $\mathrm{D}(\mathbf{5 a})$ and germacrene $\mathrm{A}(\mathbf{3 a})$ were $76 \%$ and $40 \%$, respectively (Fig. 2).

Incubations of FDP analogues with GAS: fluorinated germacrene A analogues were obtained from incubations of GAS with 6F-FDP (1b) and 14F-FDP (1d) (Fig. 2). These compounds were identified by GC-MS through co-elution with authentic material previously isolated from experiments with aristolochene synthase from Penicillium roqueforti (PR-AS, see ESI $\dagger$ ). ${ }^{4 c}$

Upon incubation with GAS, 10F-FDP (1c) produced efficiently ( $30 \%$ rel.) a single fluorinated hydrocarbon that co-eluted in the GC-MS column with an authentic sample of $\alpha$-10F-humelene, a known compound prepared previously using $\delta$-cadinene synthase (DCS). ${ }^{4 d}$ This result demonstrates that with diphosphate 1c, both enzymes are able to catalyze an anti-Markovnikov 1,11-macrocyclisation via $\pi$-donation from the vinylic fluorine atom into the distal C10,C11-double bond of $\mathbf{1 c}$.

14Me-FDP (1f) was also readily turned over by GAS yielding a mixture of at least seven hydrocarbons. The major product (retention time 28.5 min, approx. $>50 \%$ total hydrocarbons, ESI $\dagger$ ) underwent a thermal rearrangement, thereby suggesting a germacrene A analogue as the major enzymatic product from substrate 1f. In addition, in a parallel study with PR-AS, the same compound mixture was generated from $\mathbf{1 f}$ (ESI + ). Purification by preparative TLC and subsequent ${ }^{1} \mathrm{H}-\mathrm{NMR}$ analysis of this sample unambiguously confirmed the structure of the major GAS- and PR-AS-generated products as $14 \mathrm{Me}-$ germacrene A (3f). Hydrocarbon $3 \mathbf{f}$ displayed the well documented conformational flexibility exhibited by germacrene A (3a). ${ }^{12}$ Indeed, ${ }^{1} \mathrm{H}-\mathrm{NMR}$ spectra comparisons $(\mathrm{ESI} \dagger)$ with those previously obtained for (3a) at different temperatures ${ }^{12}$ suggested that $\mathbf{3 d}$ exists as an interconverting mixture of the same three conformers observed for 3a, albeit with different relative populations $(\mathrm{ESI} \dagger)$. As with the parent hydrocarbon $\mathbf{3 a}$, the most abundant conformation corresponds to the "crossed up-up' (UU) configuration, ${ }^{12}$ but in contrast to $3 \mathbf{a}$, the combined 'parallel down-up' DU and 'up-down' UD conformations of $\mathbf{3 f}$ dominates $(61 \%)$ over the individually more stable (i.e. more abundant) UU conformation (39\%). The present conformational distribution relates to the apparent increase in steric bulk on $\mathrm{C} 14$ of $3 \mathbf{f}$ relative to $\mathbf{3 a}$, which likely raises the energy of the $\mathrm{UU}$ conformer with respect to the UD and DU conformations. For a diagram and further explanation regarding the conformations of $3 \mathbf{f}$ see ESI. $\dagger$

Incubations of FDP analogues with GDS: 6F-FDP (1b) and 14Me-FDP (1f) were turned over efficiently by GDS each giving a single product displaying the more abundant and stable $[\mathrm{M}-43]^{+}$fragment in their $\mathrm{EI}^{+}$-MS spectra, which is characteristic of germacrene $\mathrm{D}(\mathbf{5 a})$ through loss of the isopropyl group within the MS-detector. Indeed, preparative incubations followed by direct ${ }^{1} \mathrm{H}$ - and ${ }^{19} \mathrm{~F}-\mathrm{NMR}$ spectroscopy analysis confirmed their identity as the expected germacrene $\mathrm{D}$ analogues $(\mathrm{ESI} \dagger)$.

Two products $(10 \%$ rel.) in an approximate $3: 1$ ratio were observed in the pentane extracts from incubation of 10F-FDP (1c) with GDS (ESI $\dagger)$. GC-MS analysis of a mixture of 10-fluoro-farnesenes, prepared in a previous study, ${ }^{4 d}$ unambiguously identified the major product as $(E)-\beta-10 \mathrm{~F}$-farnesene (ESI $\dagger)$. Interestingly, the minor component $(25 \%)$ of this mixture was also identified by co-elution as the fluorinated $\alpha-10 \mathrm{~F}-\mathrm{humulene}$ previously observed in incubations of $\mathbf{1} \mathbf{c}$ with GAS or DCS. ${ }^{4 d}$

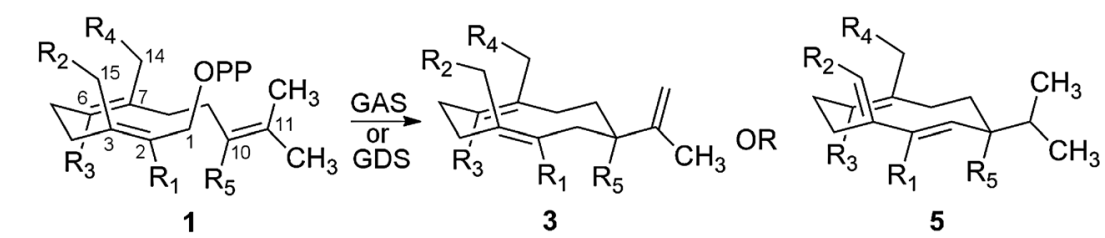

\begin{tabular}{|c|c|c|c|c|c|c|c|c|c|}
\hline \multirow{3}{*}{$\begin{array}{c}\text { Substrate } \\
\mathbf{1 a}\end{array}$} & \multicolumn{5}{|c|}{ Substituent } & \multicolumn{2}{|c|}{3 GAS } & \multicolumn{2}{|c|}{5 GDS } \\
\hline & $\mathrm{R}_{1}$ & $\mathrm{R}_{2}$ & $\mathrm{R}_{3}$ & $\mathrm{R}_{4}$ & $\mathrm{R}_{5}$ & Conv. & rel. $^{a}$ & Conv. & rel. $^{b}$ \\
\hline & $\mathrm{H}$ & $\mathrm{H}$ & $\mathrm{H}$ & $\mathrm{H}$ & $\mathrm{H}$ & 40 & 100 & 76 & 100 \\
\hline $1 b$ & $\mathrm{H}$ & $\mathrm{H}$ & $F$ & $\mathrm{H}$ & $\mathrm{H}$ & 30 & 74 & 38 & 50 \\
\hline 1d & $\mathrm{H}$ & $\mathrm{H}$ & $\mathrm{H}$ & $F$ & $\mathrm{H}$ & 19 & 47 & 33 & 43 \\
\hline $1 \mathrm{e}$ & $\mathrm{H}$ & $F$ & $\mathrm{H}$ & $\mathrm{H}$ & $\mathrm{H}$ & & & 37 & 49 \\
\hline $1 f$ & $\mathrm{H}$ & $\mathrm{H}$ & $\mathrm{H}$ & $\mathrm{Me}$ & $\mathrm{H}$ & 23 & 57 & 45 & 59 \\
\hline
\end{tabular}

Fig. 2 Incubations of modified FDP (1b, $\mathbf{d}-\mathbf{f})$ under optimized conditions: ${ }^{\mathrm{a}} \mathrm{GAS}(6 \mu \mathrm{M}), \mathrm{Mg}^{2+}(5 \mathrm{mM})$ and FDP $(0.35 \mathrm{mM}){ }^{\mathrm{b}} \mathrm{GDS}(12 \mu \mathrm{M})$, $\mathrm{Mg}^{2+}(10 \mathrm{mM})$ and FDP $(0.35 \mathrm{mM})$. Conversions were determined by GC-FID (ESI $\dagger$ ) in pentane. Relative conversions (rel.) denote percentage with respect to $\mathbf{1 a}$. 
Both 15F-FDP (1e) and 14F-FDP (1d) were converted by GDS as judged by GC-MS to a well-defined product under analytical conditions; each product (5e and $\mathbf{5 d}$ ) displayed the major $[\mathrm{M}-43]^{+}$-fragment suggesting that they were indeed germacrene D derivatives. However, prolonged preparative incubations led to the formation of a second product apparently arising from the initial GDS-generated product (ESI + ). Although the presence of this minor product hampered a full NMR interpretation of the spectrum of the original enzymatic product (5e), the observation $\left({ }^{1} \mathrm{H}\right.$ NMR, $\left.500 \mathrm{MHz}\right)$ of a relatively downfield (approx. $2 \mathrm{ppm}$ with respect to 5a) wide doublet at $\delta_{\mathrm{H}}=6.53 \mathrm{ppm}\left({ }^{2} J_{\mathrm{H}-\mathrm{F}}=86.0 \mathrm{~Hz}, \mathrm{C}=\mathrm{C} H \mathrm{~F}\right)$ instead of the diagnostic broad doublet at $\delta_{\mathrm{H}}=4.77 \mathrm{ppm}\left({ }^{2} J_{\mathrm{H}-\mathrm{H}}=\right.$ $13.0 \mathrm{~Hz}, \mathrm{C}=\mathrm{CHH}$, exo methylene group) of $\mathbf{5} \mathbf{a}^{13 c}$ (ESI $\dagger$ ) is consistent with the major product being $15 \mathrm{~F}$-germacrene D (5e, Fig. 2). Surprisingly, the ${ }^{19}$ F-NMR spectrum of this mixture displayed three absorbances, two identical doublets $\left({ }^{2} J_{\mathrm{H}-\mathrm{F}}=86.0 \mathrm{~Hz}\right)$ at $\delta_{\mathrm{F}}=-136.1$ (minor) and -138.6 (major) ppm, respectively, plus a downfield triplet $\left({ }^{2} J_{\mathrm{H}-\mathrm{F}}=46.0 \mathrm{~Hz}\right)$ at $-184.8 \mathrm{ppm}$ due to the very minor peak observable by GC-MS. Thus, the major (and only) enzymatic product is most likely produced by GDS as a mixture of two geometric 15F-germacrene D isomers (5e) (Fig. 2) that is not resolved by GC-MS. This observation implies that the corresponding tightly bound carbocation (4e) possesses sufficient mobility within the active site of GDS to allow a not completely specific proton-loss to generate the observed isomeric mixture of $\mathbf{5 e}$.

In summary, the results presented here provide insight into aspects of the reaction mechanisms employed by GAS and GDS and describe a general chemoenzymatic approach for the synthesis of non-natural terpenoids that are otherwise not easily accessible by classical chemical synthesis or synthetic biology. Indeed, these results show that GAS and GDS can turn over a variety of modified FDPs to germacrene A and D analogues often with synthetically acceptable conversions and in sufficient amounts for biological testing as semiochemicals.

This work was supported by the United Kingdom's Biotechnology and Biological Sciences Research Council through grants $\mathrm{BB} / \mathrm{G} 003572 / 1$ and $\mathrm{BB} / \mathrm{H} 01683 \mathrm{X} / 1$ and by Cardiff University. We thank Dr Rob Jenkins, Cardiff University, for assistance with NMR spectroscopy.

\section{Notes and references}

1 J. S. Glasby, Encyclopedia of Terpenoids, Wiley, Chichester, 1982. 2 A. J. Minnaard, J. B. P. A. Wijnberg and A. De Groot, Tetrahedron, 1999, 55, 2115-2146.

3 (a) N. Misawa, Curr. Opin. Biotechnol., 2011, 22, 627-633; (b) P. A. Ajikumar, et. al., Science, 2010, 330, 70-74; (c) P. J. Westfall, et. al., Proc. Natl. Acad. Sci. U. S. A., 2012, 109, 111-118.
4 (a) D. J. Miller, F. L. Yu and R. K. Allemann, ChemBioChem, 2007, 8, 1819-1825; (b) D. J. Miller, F. L. Yu, N. J. Young and R. K. Allemann, Org. Biomol. Chem., 2007, 5, 3287-3298; (c) D. J. Miller, F. L. Yu, D. W. Knight and R. K. Allemann, Org. Biomol. Chem., 2009, 7, 962-975; (d) J. A. Faraldos, D. J. Miller, V. Gonzalez, Z. Yoosuf-Aly, O. Cascón, A. Li and R. K. Allemann, J. Am. Chem. Soc., 2012, 134, 5900-5908.

5 (a) J. P. Noel, N. Dellas, J. A. Faraldos, M. Zhao, B. A. Hess, L. Smentek, R. M. Coates and P. E. O'Maille, ACS Chem. Biol., 2010, 5, 377-392; (b) J. A. Faraldos, Y. X. Zhao, P. E. O'Maille, J. P. Noel and R. M. Coates, ChemBioChem, 2007, 8, 1826-1833; (c) Y. Jin, D. C. Williams, R. Croteau and R. M. Coates, J. Am. Chem. Soc., 2005, 127, 7834-7842; (d) D. E. Cane, G. H. Yang, Q. Xue and J. H. Shim, Biochemistry, 1995, 34, 2471-2479; (e) D. E. Cane and Y. S. Tsantrizos, J. Am. Chem. Soc., 1996, 118, 10037-10040.

6 (a) D. J. Miller and R. K. Allemann, Nat. Prod. Rep., 2012, 29, 60-71; (b) E. Y. Shishova, F. L. Yu, D. J. Miller, J. A. Faraldos, Y. X. Zhao, R. M. Coates, R. K. Allemann, D. E. Cane and D. W. Christianson, J. Biol. Chem., 2008, 283, 15431-15439; (c) F. L. Yu, D. J. Miller and R. K. Allemann, Chem. Commun., 2007, 40, 4155-4157; (d) H. A. Gennadios, V. Gonzalez, L. Di Costanzo, A. Li, F. L. Yu, D. J. Miller, R. K. Allemann and D. W. Christianson, Biochemistry, 2009, 48, 6175-6183; (e) J. A. Aaron, X. Lin, D. E. Cane and D. W. Christianson, Biochemistry, 2010, 49, 1787-1797; (f) R. P. McAndrew, P. P. Peralta-Yahya, A. De Giovanni, J. H. Pereira, M. Z. Hadi, J. D. Keesling and P. D. Adams, Structure, 2011, 19, 1876-1884; $(g)$ J. A. Faraldos, A. K. Antonczak, V. González, R. Fullerton, E. M. Tippmann and R. K. Allemann, J. Am. Chem. Soc., 2011, 133, 13906-13909.

7 (a) D. W. Christianson, Chem. Rev., 2006, 106, 3412-3442; (b) M. Koksal, Y. H. Jin, R. M. Coates, R. Croteau and D. W. Christianson, Nature, 2011, 469, 116-120; (c) M. Koksal, H. Y. Hu, R. M. Coates, R. J. Peters and D. W. Christianson, Nat. Chem. Biol., 2011, 7, 431-433.

8 (a) J. A. Faraldos, B. Kariuki and R. K. Allemann, J. Org. Chem., 2010, 75, 1119-1125; (b) J. A. Faraldos and R. K. Allemann, Org. Lett., 2011, 13, 1202-1205; (c) J. A. Faraldos, B. M. Kariuki and R. M. Coates, Org. Lett., 2011, 13, 836-839.

9 (a) L. S. Vedula, Y. X. Zhao, R. M. Coates, T. Koyama, D. E. Cane and D. W. Christianson, Arch. Biochem. Biophys., 2007, 466, 260-266; (b) Z. Yoosuf-Aly, J. A. Faraldos, D. J. Miller and R. K. Allemann, Chem. Commun., 2012, 48, 7040-7042.

10 (a) W. S. Bowers, C. Nishino, M. W. Nielson, M. E. Montgomery and L. R. Nault, Science, 1977, 196, 680-681; (b) R. Mozuraitis, M. Stranden, M. I. Ramirez, A. K. Borg-Karlson and H. Mustaparta, Chem. Sens., 2002, 27, 505-509; (c) T. J. A. Bruce, et. al., Pest Manage. Sci., 2005, 61, 1115-1121; (d) M. A. Birkett, et. al., Phytochemistry, 2008, 69, 1710-1715.

11 S. L. Schreiber and R. C. Hawley, Tetrahedron Lett., 1985, 26, 5971-5974.

12 J. A. Faraldos, S. Wu, J. Chappell and R. M. Coates, Tetrahedron, 2007, 63, 7733-7742.

13 (a) I. Prosser, A. L. Phillips, S. Gittings, M. J. Lewis, A. M. Hooper, J. A. Pickett and M. H. Beale, Phytochemistry, 2002, 60, 691-702; (b) C. O. Schmidt, H. J. Bouwmeester, S. Franke and W. A. Konig, Chirality, 1999, 11, 353-362; (c) N. Bulow and W. A. Koning, Phytochemistry, 2000, 55, 141-168.

14 M. J. Calvert, P. R. Ashton and R. K. Allemann, J. Am. Chem. Soc., 2002, 124, 11636-11641.

15 A. M. Adio, Tetrahedron, 2009, 65, 1533-1552. 